\title{
Trans-Mesh Method and Its Application to Simulations of Incompressible Fluid-Rigid Bodies Interaction*
}

\author{
Shinichi ASAO ${ }^{* *}$, Kenichi MATSUNO ${ }^{* * *}$ and Masashi YAMAKAWA ${ }^{* * *}$ \\ ** Department of Mechanical Engineering, College of Industrial Technology \\ 1-27-1 Amagasaki, Hyogo, 661-0047, JAPAN \\ E-mail: asao@cit.sangitan.ac.jp \\ *** Department of Mechanical System Engineering, Kyoto Institute of Technology \\ Matsugasaki, Sakyo-ku, Kyoto, 606-8585, JAPAN
}

\begin{abstract}
In this paper, a Trans-mesh method is presented for simulating three-dimensional incompressible fluid-rigid body interaction with collisions. In the Trans-mesh method, the bodies can move freely in a main mesh that covers the entire flow field. The method is constructed based on the four-dimensional control volume in space-time unified domain such that the method assures to be divergence-free in the space-time unified domain and thus satisfies both the physical and geometrical conservation laws simultaneously. First of all, it is confirmed that the present method satisfies the geometric conservation law. Next, we did calculations for a single sphere settling under gravity in the stationary fluid to evaluate the present method. The method was applied to a flow around bodies driven by a flow in a square duct and the unsteady behavior of the flow is shown. The results indicate that this method is promising in such simulations.
\end{abstract}

Key words : Numerical Method, Incompressible Flow, Moving Mesh, Finite Volume Method, Interaction Simulation

\section{Introduction}

Unsteady flow is currently a challenging problem in computational fluid dynamics. To solve it, it is very important to calculate a moving boundary problem. In particular, cases in which bodies move in fluid while colliding with each other and/or obstacles are intriguing from an engineering or biomechanics perspective. Examples include particles flowing through a permeable film, and blood flowing in the blood vessels through valves and thrombi.

When we simulate such a flow field, we encounter some problems that must be overcome. One problem involves a mesh system. When a body moves in the flow field, it is hard to adjust the motion of the body using a conventional single body-fitted grid system. One of the most popular methods used to solve such a moving boundary problem is the overset grid method ${ }^{(1)}$, where a sub-grid placed around the moving body moves in the main grid. However, the overset grid method generally does not satisfy conservation laws rigorously due to a non-physical interpolation error. From the viewpoint of accurate computation, a body-fitted single-grid system is desirable, but is difficult in cases where bodies travel a long distance in the flow field because of the resulting highly skewed mesh.

To overcome these problems, we proposed the Moving Embedded-grid method ${ }^{(2),(3)}$. This method patches a local mesh generated around a body in a stationary main mesh and shifts the embedded local mesh in any direction through the main mesh while eliminating front-side meshes, adding back-side meshes with satisfying both physical and geometric conservation laws. As a flow solver, we modified the Moving-grid Finite-volume method ${ }^{(4)}$ and used it in the Moving Embedded-grid method.

To advance this idea further, we propose a Trans-mesh method for three-dimensional

*Received 16 Sep., 2011 (No. 11-0562) [DOI: 10.1299/jcst.5.163]

Copyright @ 2011 by JSME 
space. This new method does not use a local mesh as an embedded-grid or overset grid. It is therefore easy to deal with a contingence of bodies because no local mesh is used. The method shifts the bodies in any direction through the main mesh while eliminating front-side meshes of the moving bodies and adding back-side meshes that satisfy both physical and geometric conservation laws.

The purpose of this paper is to introduce the Trans-mesh method. First, we confirm that the present Trans-mesh method satisfies the geometric conservation law. Then we describe how the method was applied to a single sphere settling under gravity in order to validate the reliability of the method and calculate the fluid-body interaction. Then the interactive movement of multiple spheres driven by a flow through a square duct is explained.

\section{Trans-mesh Method}

\subsection{Concept of Trans-mesh method}

In the Trans-mesh method, a body moves freely in a stationary mesh while mesh planes are added, removed, and/or shifted in the main mesh, as illustrated in Fig. 1. The front mesh plane of the moving body is eliminated from the main mesh in order to prevent the mesh from folding because of the reduced mesh spacing that occurs due to the movement of the body. Moreover, a new mesh plane is added between the rear plane of the moving body and the main mesh in order to maintain the maximum allowable mesh spacing. At the same time, the cells existing and connecting between the main mesh and the side of the moving body are skewed due to the movement of the body. Thus, reconnection or exchange of mesh lines between the moving body and the main mesh is necessary. Hence, the present method essentially includes three inevitable procedures: addition and elimination of mesh planes as well as changes in the connecting relationships of structured mesh lines.

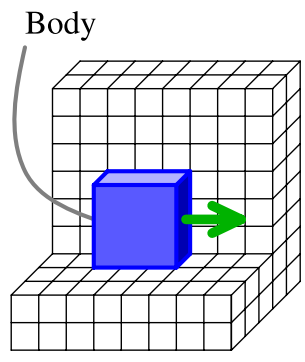

Step 1

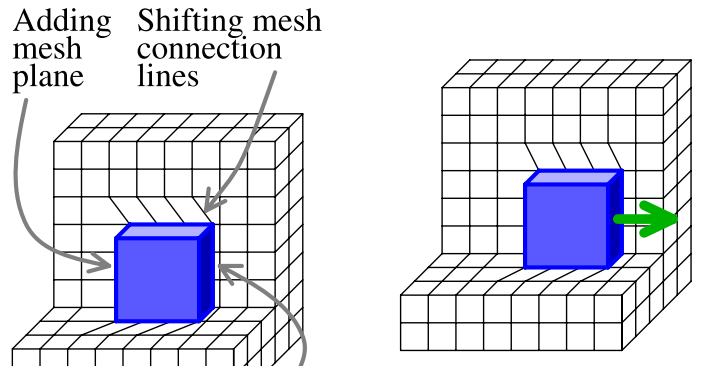

Step 3

\section{Step $2 \quad$ mesh plane}

Fig. 1 Concept of Trans-mesh method.

\subsection{Governing equations}

Governing equations are the continuity equation and the incompressible Navier-Stokes equations. These are written as follows:

$$
\begin{aligned}
& \begin{array}{l}
\nabla \cdot \boldsymbol{q}=0, \\
\frac{\partial \boldsymbol{q}}{\partial t}+\frac{\partial \boldsymbol{E}_{a}}{\partial x}+\frac{\partial \boldsymbol{F}_{a}}{\partial y}+\frac{\partial \boldsymbol{G}_{a}}{\partial z}= \\
\quad-\left(\frac{\partial \boldsymbol{E}_{p}}{\partial x}+\frac{\partial \boldsymbol{F}_{p}}{\partial y}+\frac{\partial \boldsymbol{G}_{p}}{\partial z}\right)+\left(\frac{\partial \boldsymbol{E}_{v}}{\partial x}+\frac{\partial \boldsymbol{F}_{v}}{\partial y}+\frac{\partial \boldsymbol{G}_{v}}{\partial z}\right)
\end{array}
\end{aligned}
$$

where $\boldsymbol{q}$ is a velocity vector, $\boldsymbol{E}_{a}, \boldsymbol{F}_{a}$, and $\boldsymbol{G}_{a}$ are advection flux vectors in the $x, y$, and $z$ directions, respectively, $\boldsymbol{E}_{v}, \boldsymbol{F}_{v}$, and $\boldsymbol{G}_{v}$ are viscous-flux vectors, and $\boldsymbol{E}_{p}, \boldsymbol{F}_{p}$, and $\boldsymbol{G}_{p}$ are 
pressure flux vectors. The elements of the velocity vector and flux vectors are

$$
\begin{aligned}
& \boldsymbol{q}=\left[\begin{array}{c}
u \\
v \\
w
\end{array}\right], \quad \boldsymbol{E}_{a}=\left[\begin{array}{c}
u^{2} \\
u v \\
u w
\end{array}\right], \quad \boldsymbol{F}_{a}=\left[\begin{array}{c}
u v \\
v^{2} \\
v w
\end{array}\right], \quad \boldsymbol{G}_{a}=\left[\begin{array}{c}
u w \\
v w \\
w^{2}
\end{array}\right], \\
& \boldsymbol{E}_{v}=\frac{1}{\operatorname{Re}}\left[\begin{array}{c}
u_{x} \\
v_{x} \\
w_{x}
\end{array}\right], \quad \boldsymbol{F}_{v}=\frac{1}{\operatorname{Re}}\left[\begin{array}{c}
u_{y} \\
v_{y} \\
w_{y}
\end{array}\right], \quad \boldsymbol{G}_{v}=\frac{1}{\operatorname{Re}}\left[\begin{array}{c}
u_{z} \\
v_{z} \\
w_{z}
\end{array}\right], \\
& \boldsymbol{E}_{p}=\left[\begin{array}{c}
p \\
0 \\
0
\end{array}\right], \quad \boldsymbol{F}_{p}=\left[\begin{array}{c}
0 \\
p \\
0
\end{array}\right], \quad \boldsymbol{G}_{p}=\left[\begin{array}{c}
0 \\
0 \\
p
\end{array}\right]
\end{aligned}
$$

where $u, v$, and $w$ are velocity components of the $x, y$, and $z$ directions, respectively, $p$ is pressure, and Re is the Reynolds number. The subscripts $x, y$, and $z$ indicate derivatives with respect to $x, y$, and $z$.

In this paper, we consider the combined motion of the translation and rotation of a body. The motion of the body is considered to have six degrees of freedom (6DOF). The 6-DOF rigid body equations of motion are written as follows:

$$
\begin{aligned}
& \frac{\mathrm{d} \boldsymbol{p}_{\mathrm{B}}}{\mathrm{d} t}=\boldsymbol{f}_{\mathrm{B}}, \\
& \frac{\mathrm{d} \boldsymbol{L}_{\mathrm{B}}}{\mathrm{d} t}=\boldsymbol{N}_{\mathrm{B}}
\end{aligned}
$$

where $\boldsymbol{p}_{\mathrm{B}}$ is the linear momentum vector of the body, $\boldsymbol{L}_{\mathrm{B}}$ is the angular momentum vector, $\boldsymbol{f}_{\mathrm{B}}$ is the force acting on the body, and $N_{\mathrm{B}}$ is torque. The elements of momentum and angular momentum vector are

$$
\boldsymbol{p}_{\mathrm{B}}=\left[\begin{array}{c}
m \dot{r}_{\mathrm{G} x} \\
m \dot{r}_{\mathrm{G} y} \\
m \dot{r}_{\mathrm{G} z}
\end{array}\right], \quad \boldsymbol{L}_{\mathrm{B}}=\left[\begin{array}{c}
I_{x} \dot{\theta}_{x} \\
I_{y} \dot{\theta}_{y} \\
I_{z} \dot{\theta}_{z}
\end{array}\right], \quad \boldsymbol{f}_{\mathrm{B}}=\left[\begin{array}{c}
f_{\mathrm{B} x} \\
f_{\mathrm{B} y} \\
f_{\mathrm{B} z}
\end{array}\right], \quad \boldsymbol{N}_{\mathrm{B}}=\left[\begin{array}{c}
N_{\mathrm{B} x} \\
N_{\mathrm{B} y} \\
N_{\mathrm{B} z}
\end{array}\right],
$$

where $m$ is the mass of the body, $I_{x}, I_{y}$, and $I_{z}$ are the inertia moment components of the $x, y$, and $z$ directions, $r_{\mathrm{G} x}, r_{\mathrm{G} y}$, and $r_{\mathrm{G} z}$ are the positions of the center of gravity, and $\theta_{x}, \theta_{y}$, and $\theta_{z}$ are angular position components of the axis about the body. A dot over the variable indicates derivation with respect to time.

\subsection{Moving-grid Finite-volume method}

To assure the geometric conservation laws, we adopt a control volume in the space-time unified domain $(x, y, z, t)$, which is four-dimensional in the case of three-dimensional flows. Now, Eq. (2) can be written in divergence form as,

$$
\tilde{\nabla} \cdot \tilde{\mathcal{F}}=\mathbf{0}
$$

where

$$
\begin{aligned}
& \tilde{\nabla}=\left[\begin{array}{llll}
\frac{\partial}{\partial x} & \frac{\partial}{\partial y} & \frac{\partial}{\partial z} & \frac{\partial}{\partial t}
\end{array}\right]^{T}, \quad \tilde{\mathcal{F}}=\left[\begin{array}{cccc}
\boldsymbol{E} & \boldsymbol{F} & \boldsymbol{G} & \boldsymbol{q}
\end{array}\right]^{T}, \\
& \boldsymbol{E}=\boldsymbol{E}_{a}-\boldsymbol{E}_{v}+\boldsymbol{E}_{p}, \quad \boldsymbol{F}=\boldsymbol{F}_{a}-\boldsymbol{F}_{v}+\boldsymbol{F}_{p}, \quad \boldsymbol{G}=\boldsymbol{G}_{a}-\boldsymbol{G}_{v}+\boldsymbol{G}_{p} .
\end{aligned}
$$

The present method is based on a cell-centered finite-volume method and, thus, the flow variables are defined at the center of the cell in the $(x, y, z)$ space. The control volume becomes a four-dimensional polyhedron in the $(x, y, z, t)$-domain, as schematically illustrated in Fig. 2.

We apply volume integration to Eq. (2) with respect to the control volume illustrated in Fig. 2. By using the Gauss theorem, we can write Eq. (7) in surface integral form as,

$$
\int_{\tilde{\Omega}} \tilde{\nabla} \cdot \tilde{\mathcal{F}} \mathrm{d} \tilde{\Omega}=\oint_{\tilde{S}} \tilde{\mathcal{F}} \cdot \tilde{\boldsymbol{k}} \mathrm{d} \tilde{S}=\sum_{l=1}^{8}(\tilde{\mathcal{F}} \cdot \tilde{\boldsymbol{n}})_{l}=\mathbf{0} .
$$




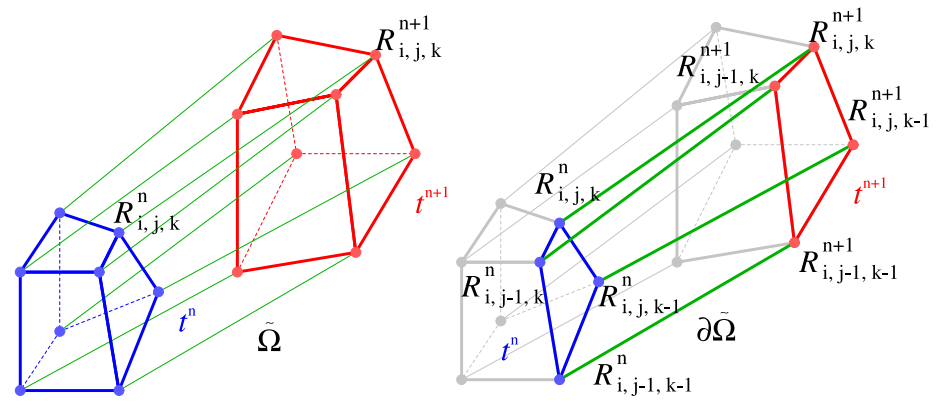

Fig. 2 Schematic view of control volume in $(x, y, z, t)$ space-time unified domain.

Here, $\tilde{\boldsymbol{k}}$ is an outward unit vector normal to the surface, $\tilde{S}$, of the octahedron control volume $\tilde{\Omega}$, in the space-time unified four-dimensional domain, and, $\tilde{\boldsymbol{n}}_{l}=\left(\tilde{n}_{x}, \tilde{n}_{y}, \tilde{n}_{z}, \tilde{n}_{t}\right)_{l},(l=1,2, \ldots 8)$ denotes the surface normal vector of the control volume and its length equal to the boundary surface area in four-dimensional $(x, y, z, t)$ space. The upper and lower boundaries of the control volume ( $l=7$ and 8$)$ are perpendicular to the $t$-axis, and, therefore, they only have an $\tilde{n}_{t}$ component, and its length corresponds to the volume of the cell in the $(x, y, z)$-space at times $t^{n}$ and $t^{n+1}$ respectively. Thus, Eq. (9) can be expressed as,

$$
\boldsymbol{q}^{n+1}\left(\tilde{n}_{t}\right)_{8}+\boldsymbol{q}^{n}\left(\tilde{n}_{t}\right)_{7}+\sum_{l=1}^{6}\left(\tilde{\mathcal{F}}^{n+1 / 2} \cdot \tilde{\boldsymbol{n}}\right)_{l}=\mathbf{0}
$$

\subsection{Formulation of Trans-mesh method}

The addition of the mesh plane is done as follows. Suppose that mesh cell $I$ at time step $n$ deforms as well as moves to a new position, and then separates into two mesh cells $I$ and $I^{\prime}$ at time step $(n+1)$, as illustrated in Fig. 3. This means that the new mesh plane A'D'H'E' is inserted or added between mesh planes ADHE and BCGF. Under this situation, we have to treat two control volumes, $I$ and $I^{\prime}$, to get solutions at time step $(n+1)$. For control volume $I$, Eq. (10) is applied, and no other procedures are necessary. For the newly appearing control volume $I^{\prime}$, we need to modify Eq. (10) taking into consideration that the volume of cell $I^{\prime}$ at time $n$ is zero. This means that $\left(\tilde{n}_{t}\right)_{7}=0$ for control volume $I^{\prime}$. Thus, the finite volume equation for $I^{\prime}$ becomes:

$$
\boldsymbol{q}^{n+1}\left(\tilde{n}_{t}\right)_{8 I^{\prime}}+\sum_{l=1}^{6}\left(\tilde{\mathcal{F}}^{n+1 / 2} \cdot \tilde{\boldsymbol{n}}\right)_{l}=\mathbf{0}
$$

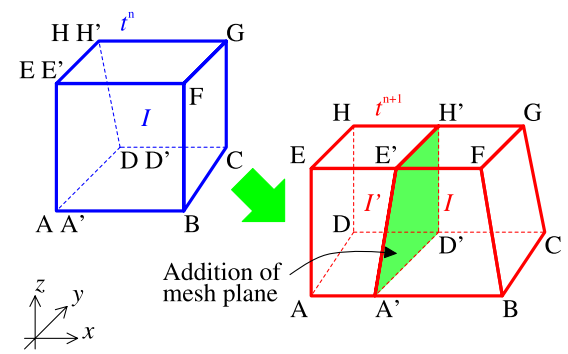

Fig. 3 Control volume for addition of mesh plane.

The elimination of the mesh plane is accomplished through merging the two related control volumes. Suppose that the mesh plane PQRS is eliminated at the $(n+1)$-time step. Then we consider that the $I^{\prime}$-th and $I$-th cells at the $n$-time step are merged at the $(n+1)$-time step, and only the $I$-th cell remains. In this situation, the control volume in the space-time 
unified domain, $(x, y, z, t)$, is as illustrated in Fig. 4. Thus, Eq. (12) replaces Eq. (10).

$$
\boldsymbol{q}_{I}^{n+1}\left(\tilde{n}_{t}\right)_{8 I}+\boldsymbol{q}_{I}^{n}\left(\tilde{n}_{t}\right)_{7 I}+\boldsymbol{q}_{I^{\prime}}^{n}\left(\tilde{n}_{t}\right)_{7 I^{\prime}}+\sum_{l=1}^{6}\left(\tilde{\mathcal{F}}^{n+1 / 2} \cdot \tilde{\boldsymbol{n}}\right)_{l}=\mathbf{0}
$$

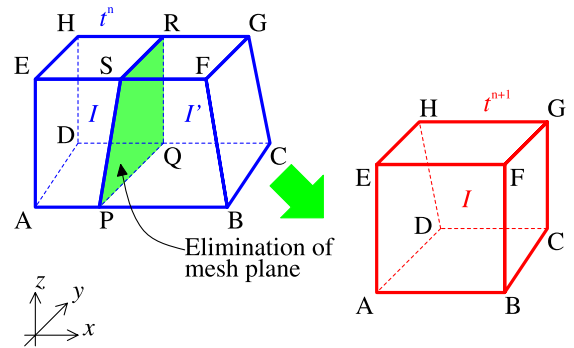

Fig. 4 Control volume for elimination of mesh plane.

To shift the mesh connection lines at the $(n+1)$-time step, the control volume between the $n$ and $(n+1)$-time steps is as shown in Fig. 5. The flux at the $l=7$ ( $n$-time step) cell is divided according to the new connection of mesh connection lines before the volume integral is applied to the control volume to calculate the $(n+1)$-time step. This is supported because in the finite-volume formulation, the value of the variable is assumed to be constant in the cell; thus, the estimation of flux at the $n$-time step is straightforward. For this situation, the flux at the $n$-time step is divided into two parts. By referring to Fig 5, we can replace Eq. (10) with Eq. (13).

$$
\left[\boldsymbol{q}^{n+1}\left(\tilde{n}_{t}\right)_{8}\right]_{B C D^{\prime} C^{\prime}}+\left[\boldsymbol{q}^{n}\left(\tilde{n}_{t}\right)_{7}\right]_{B C C^{\prime}}+\left[\boldsymbol{q}^{n}\left(\tilde{n}_{t}\right)_{7^{\prime}}\right]_{C D^{\prime} C^{\prime}}+\sum_{l=1}^{6}\left(\tilde{\mathcal{F}}^{n+1 / 2} \cdot \tilde{\boldsymbol{n}}\right)_{l}=\mathbf{0}
$$

Here, $\left[\boldsymbol{q}^{n+1}\left(\tilde{n}_{t}\right)_{8}\right]_{B C D^{\prime} C^{\prime}}$ is the flux through the control volume BCD'C', which has a hexahedron shape for the $(n+1)$ time step, $\left[\boldsymbol{q}^{n}\left(\tilde{n}_{t}\right)_{7}\right]_{B C C^{\prime}}$ is the flux through the control volume BCC', which has a triangular prism shape for the $n$ time step, and $\left[\boldsymbol{q}^{n}\left(\tilde{n}_{t}\right)_{7}\right]_{C C^{\prime} D^{\prime}}$ is the flux through the control volume CD'C', which also has a triangular prism shape for the $n$ time step.

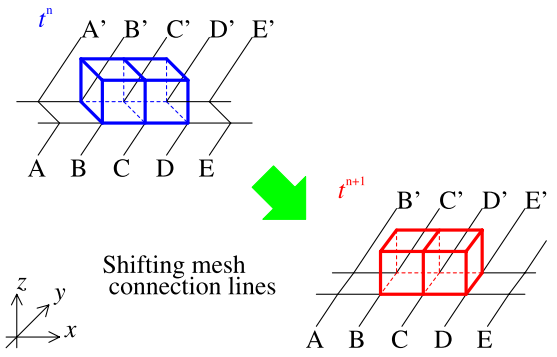

Fig. 5 Control volume for shifting the mesh connection lines.

In the present method, the addition and elimination of mesh planes are performed simultaneously when the distance between points on a regular interval mesh is as shown in Fig. 6 , and the center of the body exceeds a user-specified tolerance. In this paper, we add and eliminate the mesh if the body moves more than $1.5 \Delta x_{0}$ from the initial position of the body, where $\Delta x_{0}$ is the length that divides the computational domain by the number of mesh points.

\subsection{Solution procedure}

We apply the simplified marker and cell (SMAC) method ${ }^{(5)}$ to solve Eq. (10). Equation (10) can be solved in three stages, as follows. The equation solved in the first stage is written 


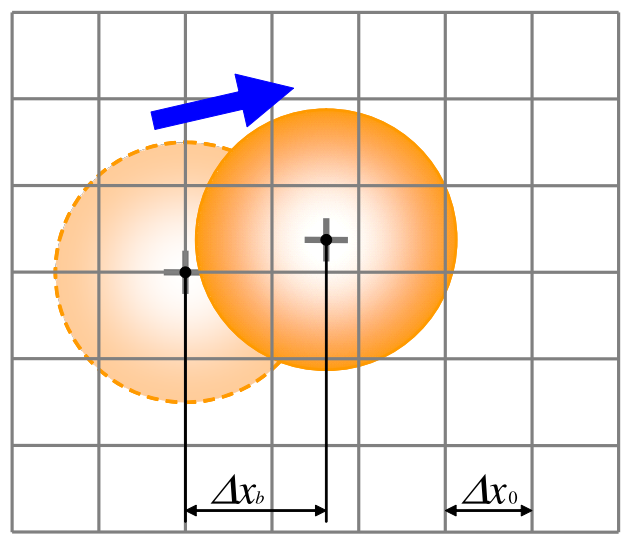

Fig. 6 Timing of adding and eliminating mesh planes.

as follows:

$$
\boldsymbol{q}^{*}\left(\tilde{n}_{t}\right)_{8}+\boldsymbol{q}^{n}\left(\tilde{n}_{t}\right)_{7}+\frac{1}{2} \sum_{l=1}^{6}\left[\left(\tilde{\mathcal{F}}^{*}+\tilde{\mathcal{F}}^{n}\right) \cdot \tilde{\boldsymbol{n}}\right]_{l}=\mathbf{0} .
$$

Here, $(*)$ denotes a tentative physical amount at the $(n+1)$-time step. The equation to be solved at the first stage contains the unknown variables at the $(n+1)$-time step in flux terms. Thus, the equation is iteratively solved using the LU-SGS method ${ }^{(6)}$. The equation to be solved at the second stage is the Poisson equation for the pressure correction. This Poisson equation is written as follows:

$$
-D^{*}\left(\tilde{n}_{t}\right)_{8}+\sum_{l=1}^{6}\left(\frac{\partial \delta p}{\partial x} \tilde{n}_{x}+\frac{\partial \delta p}{\partial y} \tilde{n}_{y}+\frac{\partial \delta p}{\partial z} \tilde{n}_{z}\right)_{l}=0,
$$

where $D=\nabla \boldsymbol{q}$, and $\delta p$ is the pressure correction. This equation is iteratively solved using the Bi-CGSTAB method ${ }^{(7)}$. A coefficient matrix of the pressure correction is a nonsymmetric matrix because a number of mesh planes do not cross at right angles. In the third stage, we calculate $\boldsymbol{q}^{n+1}$ using $\boldsymbol{q}^{*}$ and $\delta p$ solved by Eq. (14) and Eq. (15). The flux vectors are evaluated using the QUICK method, whereas the flux vectors of the pressure and viscous terms are evaluated in the central-difference-like manner.

The incompressible fluid-rigid body interaction with collision is calculated in the first step of the SMAC method. Figure 7 shows the flowchart of the fluid-body interaction. First, we calculate the force applied to the body. This force includes the force of collision. The collision force is evaluated based on Glowinski's method ${ }^{(9)}$. Next, the equation of motion for the body is calculated, and the mesh is moved. Then, the Navier-Stokes equations are calculated. If the physical amounts converge, we proceed to the third step of the SMAC method.

\section{Validation of Present Method}

\subsection{Geometric conservation laws}

It is necessary to confirm that the method satisfies the "geometric conservation law" $(\mathrm{GCL})^{(8)}$, which means that it does not disturb the uniform flow, when the mesh is moving as mesh lines are added, eliminated, and shifted. To verify that the method satisfies the GCL, we apply the present method to the uniform flow. The computational conditions are summarized as follows. The number of grid points is $21 \times 21 \times 21$ for the stationary main mesh, time spacing $\Delta t=0.01$, and the Reynolds number $\operatorname{Re}=100$. The initial condition is assumed to be $u=u_{\infty}=1.0, v=v_{\infty}=1.0, w=w_{\infty}=1.0$, and $p=p_{\infty}=1.0$ as the uniform flow. The boundary condition is fixed for $u=u_{\infty}, v=v_{\infty}$, and $w=w_{\infty}$, and pressure at the boundary is fixed for $p=p_{\infty}$. Figure 8 shows the movement of the computational mesh at $t=0,0.5,1$, and 1.5. The gray mesh is fixed and the yellow mesh is moved. The mesh plane between the gray and yellow mesh is added, eliminated, and exchanged. 


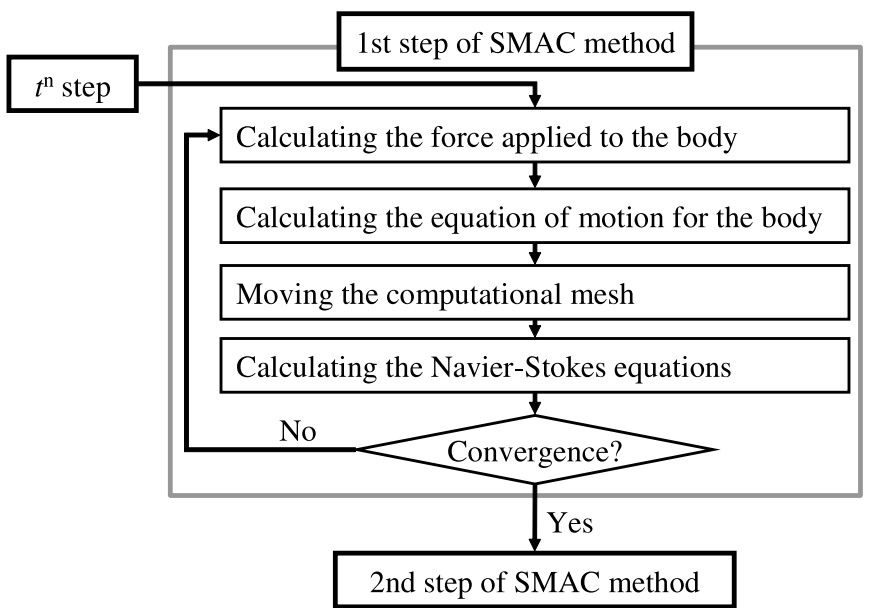

Fig. 7 Flowchart for calculation of interaction.
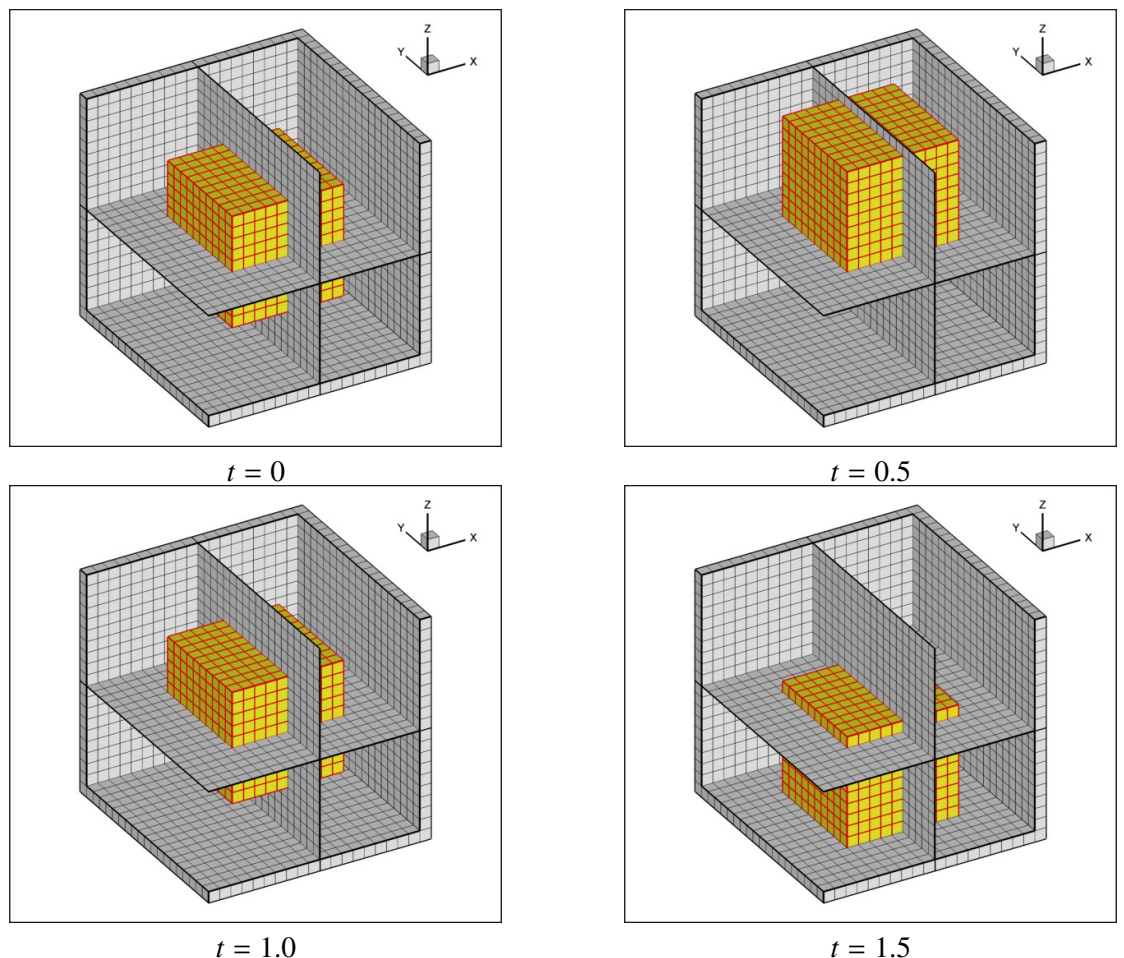

Fig. 8 Movement of computational mesh while adding, eliminating, and shifting mesh lines. 
The calculation was performed until $t=1000$. The velocity and pressure errors are defined as follows.

$$
\begin{aligned}
& \text { Error }_{\mathrm{vel}}=\max _{I, J, K} \sqrt{\frac{\Delta u_{I, J, K}^{2}+\Delta v_{I, J, K}^{2}+\Delta w_{I, J, K}^{2}}{3}}, \\
& \text { Error }_{\text {pre }}=\max _{I, J, K}\left|p_{I, J, K}-p_{\infty}\right| .
\end{aligned}
$$

Here,

$$
\Delta u_{I, J, K}=u_{I, J, K}-u_{\infty}, \quad \Delta v_{I, J, K}=v_{I, J, K}-v_{\infty}, \quad \Delta w_{I, J, K}=w_{I, J, K}-w_{\infty} .
$$
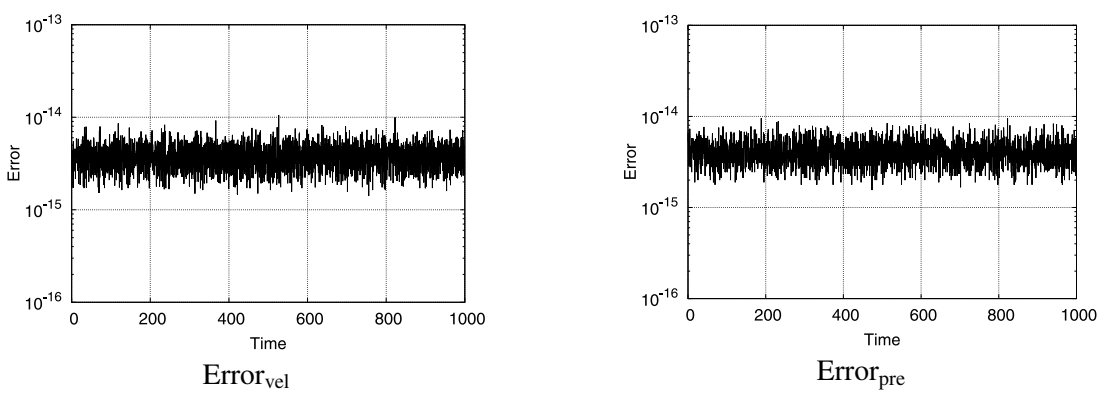

Fig. 9 Error history.

After the 1,000 time, the errors keep the order of $10^{-15}$ as shown in Fig. 9, in other words, machine zero, which means the method satisfies GCL perfectly.

\subsection{Simulations on a single sphere settling under gravity}

To evaluate the present method, we did calculations for a single sphere settling under gravity in the stationary fluid.

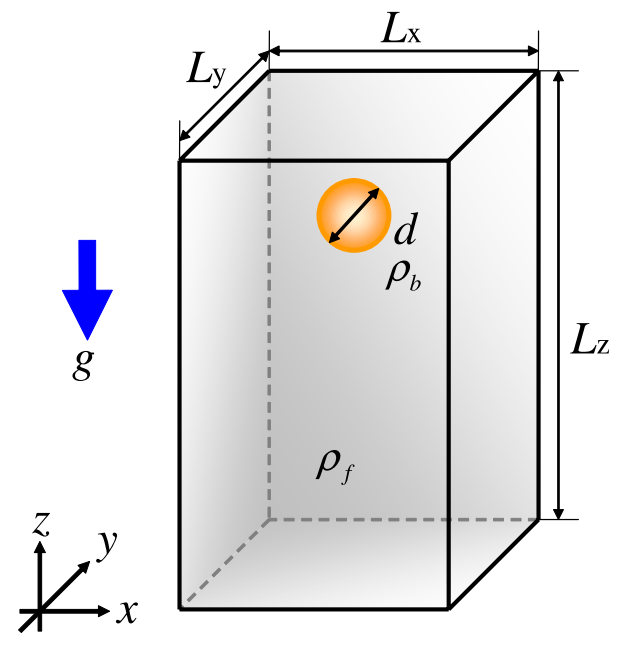

Fig. 10 Computational domain for a falling sphere.

A settling sphere with a diameter $d=15 \mathrm{~mm}$ and density $\rho_{\mathrm{b}}=1120 \mathrm{~kg} / \mathrm{m}^{3}$ was selected. The container dimensions were width $L_{x} \times$ depth $L_{y} \times$ height $L_{z}=100 \mathrm{~mm} \times 100 \mathrm{~mm} \times 160 \mathrm{~mm}$, as shown in Fig. 10. The sphere was dropped from $z=120 \mathrm{~mm}$. The Reynolds number of the settling sphere is based on the terminal velocity $w_{\infty}$ of a dropping sphere in stationary fluid. To determine $w_{\infty}$, a relation for the drag coefficient due to Abraham $^{(10)}$ is used. The four cases are defined in Table 1. 
Table 1 Setup of physical quantity

\begin{tabular}{lcccr}
\hline Case number & $\rho_{\mathrm{f}}\left[\mathrm{kg} / \mathrm{m}^{3}\right]$ & $\mu_{\mathrm{f}}\left[\mathrm{Ns} / \mathrm{m}^{2}\right]$ & $w_{\infty}[\mathrm{m} / \mathrm{s}]$ & \multicolumn{1}{c}{$\mathrm{Re}$} \\
\hline Case 1 & 970 & $373 \times 10^{-3}$ & 0.038 & 1.5 \\
Case 2 & 965 & $212 \times 10^{-3}$ & 0.060 & 4.1 \\
Case 3 & 962 & $113 \times 10^{-3}$ & 0.091 & 11.6 \\
Case 4 & 960 & $58 \times 10^{-3}$ & 0.128 & 31.8 \\
\hline
\end{tabular}

As the calculation condition, the time step size was $\Delta t=0.001$ for cases 1 and 2 , and $\Delta t=0.01$ for cases 3 and 4 . The number of the main mesh was $44 \times 44 \times 86$.

Figure 11 shows the vorticity contours of the $y$ direction $\left(\omega_{y}\right)$ and the computational mesh. We confirmed that the flow is generated by sedimentation of the sphere over time. The computational mesh holds its shape well even if the sphere moves to various positions.
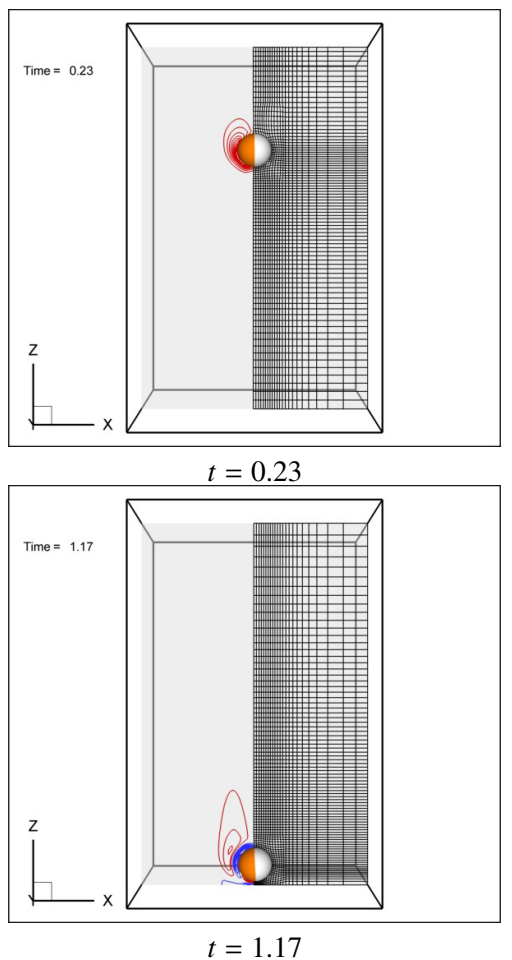
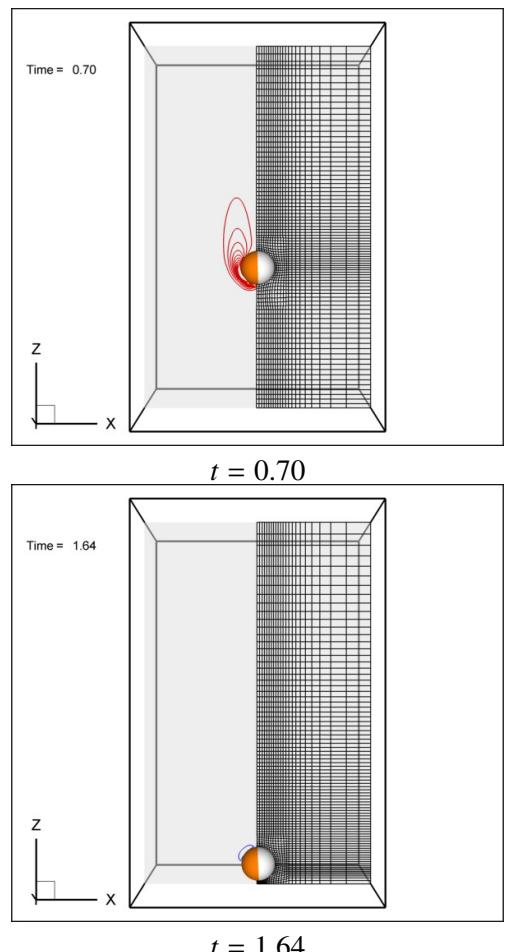

$t=1.64$

Fig. 11 Vorticity contours of $y$ direction $\left(\omega_{y}\right)$ and computational mesh for case 4.

Figure 12 compares the (a) sphere trajectories and (b) sedimentation velocities obtained using the present method with the experimental results from ten Cate et al. ${ }^{(11)}$.

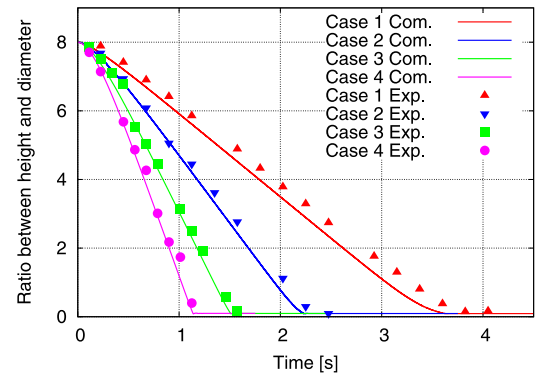

(a) Sphere trajectory

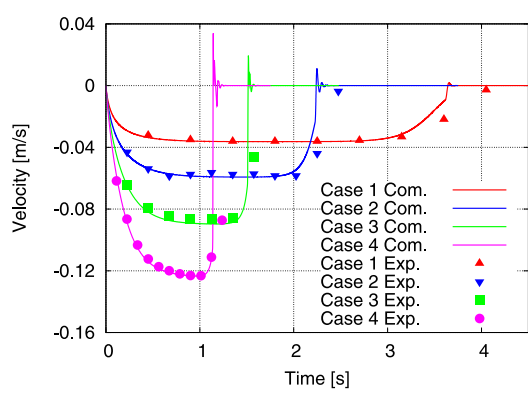

(b) Sedimentation velocity

Fig. 12 Comparisons of results obtained using body permeable-mesh method with experimental results obtained by ten Cate et al. ${ }^{(11)}$.

The sphere trajectory and sedimentation velocity are in very good agreement with the 
reference results. This indicates that the present method gave accurate solutions in the simulation.

\section{Interactive Movement of Multiple Spheres Driven by Flow in Square Duct}

\subsection{Computational conditions}

As an application of the present method, we simulated the motions of bodies, i.e., balls, driven by a flow in a square duct, as illustrated in Fig. 13.

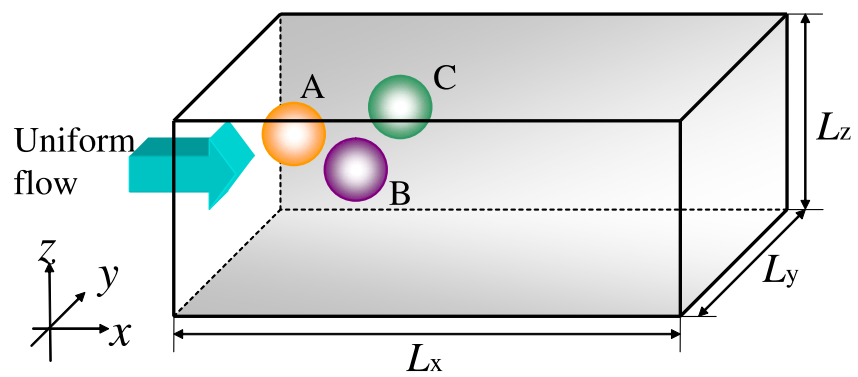

Fig. 13 Multiple spherical bodies driven by uniform flow.

The size of the entire computational domain was $L_{x}=20.0 \mathrm{D}, L_{y}=5.0 \mathrm{D}$, and $L_{z}=5.0 \mathrm{D}$, where $D$ is the diameter of the balls. The initial position $(x, y, z)$ of the balls was $(2.5 D, 2.75 D$, $3.0 D),(5.0 D, 2.5 D, 2.5 D)$, and $(6.5 D, 3.5 D, 3.5 D)$, respectively. The spherical densities of the balls were $\rho_{A}=20 \rho_{f}, \rho_{B}=40 \rho_{f}$, and $\rho_{C}=60 \rho_{f}$, respectively where $\rho_{f}$ is the density of fluid. A semi-steady flow around the fixed balls in a uniform flow was used as the initial condition. For the calculation condition, the Reynolds number was 40, and the time step size was $\Delta t=0.01$. The number of the main mesh was $201 \times 71 \times 71$.

\subsection{Numerical results}

The pressure contours and velocity vectors are shown on the left side of Fig. 14, and the rotating balls and the streamlines are shown on the right side. The colors of the streamlines indicate the magnitude of the velocity. The figure shows that smooth pressure distributions and streamlines were obtained at all times. The velocity vectors and pressures vary according to the locations of the balls. Figure 15 shows the time-history of a travel speed of the balls. The ball $\mathrm{B}$ is accelerated by colliding with the ball A at $t=17$. The ball A collides with the ball $\mathrm{C}$ at $t=26$, and it collides with the walls at $t=30$. When the ball A collides with each other and with the walls, the speed of $\mathrm{A}$ is reduced for a moment. The ball A collides with the ball $\mathrm{C}$ again at $t=34$.

\section{Concluding Remarks}

This paper introduced the Trans-mesh method and described its application to the motion of spherical bodies driven by a flow in a square duct. The Trans-mesh method was shown to satisfy the physical and geometric conservation laws simultaneously. The method was applied to a single sphere settling under gravity in order to validate the reliability of the method and the calculation of fluid-body interaction. The sphere trajectory and sedimentation velocity were found to be in very good agreement with other reported results. We also simulated the motion of bodies driven by flow in a square duct. The results indicated that physically meaningful flows were obtained. Therefore, we confirmed that the Trans-mesh method is useful for simulating the interaction of incompressible fluid-rigid bodies with collisions.

This work was supported by the "Science Research Promotion Fund (2010)" from the Promotion and Mutual Aid Corporation for Private Schools of Japan. 


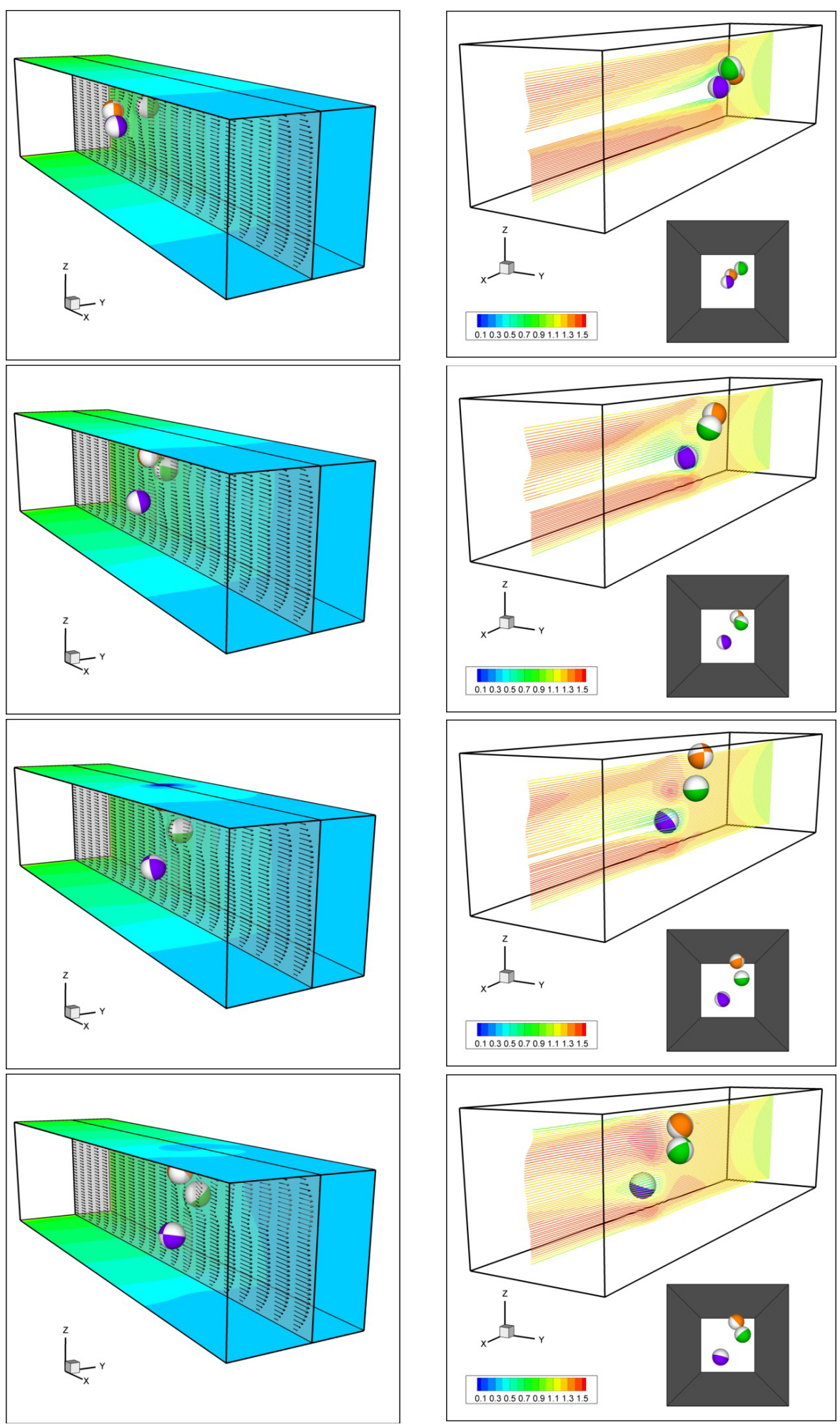

Fig. 14 Pressure contours and velocity vectors (left), and streamlines (right) at time $t=17,26,30$, and 34 . 


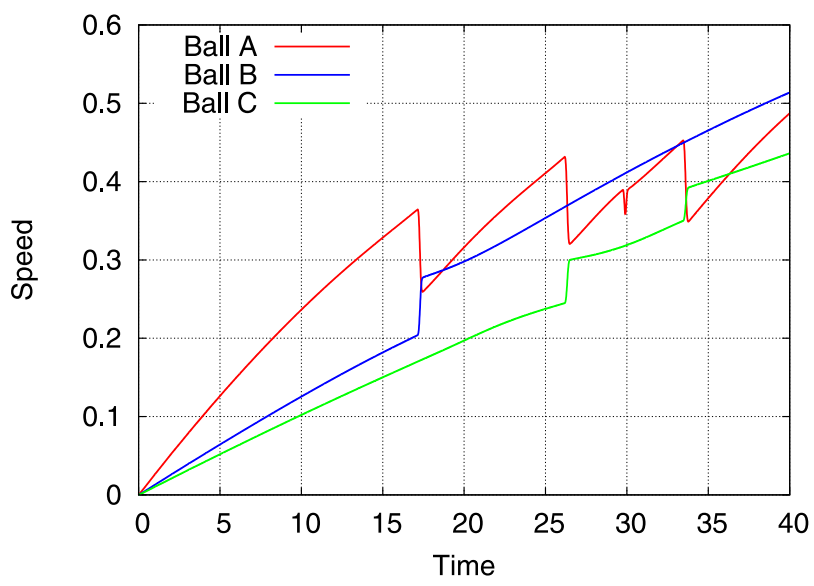

Fig. 15 Time-history of a travel speed of the balls.

\section{References}

( 1 ) Steger, J. L., Dougherty, F. C., and Benek, J. A.: A Chimera Grid Scheme; Advances in Grid Generation, American Society of Mechanical Engineers, Fluids Engineering Division, Vol. 5, (1983), pp. 55-70.

( 2 ) Asao, S. and Matsuno, K.: A Moving Embedded-Grid Method for Simulation of Unsteady Incompressible Flows with Boundaries Traveling Long Distances, JSME, Vol. 74-737 B, (2008), pp. 25-33.

( 3 ) Asao, S. and Matsuno, K.: Three-Dimensional Moving Embedded-Grid Method for the Simulation of Flows Driven by Multiple Obstacles Traveling Long Distances, JSME, Vol. 75-755 B, (2009), pp. 1379-1386.

( 4 ) Matsuno, K., Mihara, K., and Satofuka, N.: A Moving-Mesh Finite-Volume Scheme for Compressible Flows, Computational Fluid Dynamics 2000, Springer, (2001), pp. 705-710.

( 5 ) Amsden, A. A. and Harlow, F. H.: A Simplified MAC Technique for Incompressible Fluid Flow Calculations, J. Comp. Phys, Vol. 6, (1970), pp. 322-325.

( 6 ) Yoon, S. and Jameson, A.: Lower-Upper Symmetric-Gauss-Seidel Method for the Euler and Navier-Stokes Equations, AIAA J., Vol. 26, (1988), pp. 1025-1026.

( 7 ) Van der Vorst, H. A.: Bi-CGSTAB: A Fast and Smoothly Converging Variant of Bi-CG for the Solution of Nonsymmetric Linear Systems, SIAM Journal on Scientific Computing, Vol. 13-2, (1992), pp. 631-644.

( 8 ) Zhang, H., Reggio, M., Trepanier, J. Y., and Camarero, R.: Discrete Form of the GCL for Moving Meshes and its Implementation in CFD Schemes, Computers and Fluids, Vol. 22, (1993), pp. 9-23.

( 9 ) Glowinski, R., Pan, T.-W., Hesla, T. I., and Joseph, D. D.: A Distributed Lagrange Multiplier/Fictitious Domain Method for Particulate Flows, International Journal of Multiphase Flow, Vol. 25, (1999), pp. 755-794.

(10) Abraham, F. F.: Functional Dependence of Drag Coefficient of a Sphere on Reynolds number, Physics of Fluids, Vol. 13, (1970), pp. 2194-2195.

(11) Cate, A ten., Nieuwstad, C. H., Derksen, J. J., and Van den Akker, H. E. A.: Particle Imaging Velocimetry Experiments and Lattice-Boltzmann Simulations on a Single Sphere Settling under Gravity, Physics of Fluids, Vol. 14, (2002), pp. 4012-4025. 